\title{
Tunneling and Point Contact Investigations of $\mathrm{La}_{1.85} \mathrm{Sr}_{0.15} \mathrm{CuO}_{4}$
}

\author{
P. Leiderer and R. Feile \\ Institut für Physik, Johannes-Gutenberg-Universität, Mainz, \\ Federal Republic of Germany \\ B. Renker and D. Ewert \\ Institut für Nukleare Festkörperphysik, Kernforschungszentrum Karlsruhe, \\ Federal Republic of Germany
}

Received April 21, 1987

\begin{abstract}
The high- $T_{c}$ superconductor $\mathrm{La}_{1.85} \mathrm{Sr}_{0.15} \mathrm{CuO}_{4}$ was investigated by means of point contact and tunneling measurements on small-sized contacts. We find different values for the energy gap at different points of the samples. The ratio $2 \Delta / k_{B} T_{c}$ ranges from about 3 to 6 . For some point contacts we observe a clear signature of a supercurrent. Data for the temperature dependence of the critical current are presented.
\end{abstract}

\section{Introduction}

Among the new high- $T_{c}$ superconductors discovered by Bednorz and Müller [1] and subsequently developed further by $\mathrm{Chu}$ and coworkers [2] and a rapidly growing number of other groups [3-6], $\mathrm{La}_{1.85} \mathrm{Sr}_{0.15} \mathrm{CuO}_{4}$ is one of the prototypes. In order to characterize such materials, numerous investigations are presently under way. These include also tunneling and point contact measurements, which are supposed to elucidate in particular the microscopic properties of these new materials. In such experiments the current-voltage characteristic of a small-sized contact between two pieces of the superconducting sample is studied. Depending on the distance between the two electrodes low or high impedance junctions are obtained, which allow to measure critical currents and the energy gap of the superconductor, respective1y. A preliminary report of our results has already been presented earlier [7].

\section{Experimental Procedures}

The samples were prepared at the KfK Karlsruhe by a solid state reaction of the appropriate metal oxides. The material was pressed into pellets and sintered at $1050{ }^{\circ} \mathrm{C}$ for several hours and subsequently an- nealed at $900^{\circ} \mathrm{C}$ for a few days. A detailed description of the sample preparation is given elsewhere [6]. The surface of the samples appeared irregular and rugged under the microscope, and not very well suited for point contact studies. Nevertheless it was possible to prepare contacts which, once formed, yielded stable and reproducible results. This was achieved by mounting two pieces of the same material close to each other on a simple scanning device. It consists of two piezo-electric bimorphs which can be moved perpendicular to each other. One is used to adjust the distance between the samples and the other to displace them laterally. Thus different spots on the samples can be tested.

The resistance of the contacts could be varied from $10 \Omega$ to $10^{6} \Omega$ in the normal state. However, when the two pieces of the sample were brought into contact at liquid helium temperatures the resistance was in the $\mathrm{k} \Omega$ range even if the two pieces touched. The junctions could then be optimized by passing a short current pulse through the contact, which apparently cleaned the contact area.

We first show the results of high resistance contacts (i.e. a relatively large contact spacing). The $I-U$ characteristics were determined with an $\mathrm{AC}$ method using a frequency of about $100 \mathrm{~Hz}$. Both the direct signal and the electronically differentiated signal were measured and averaged about a hundred times. We found 

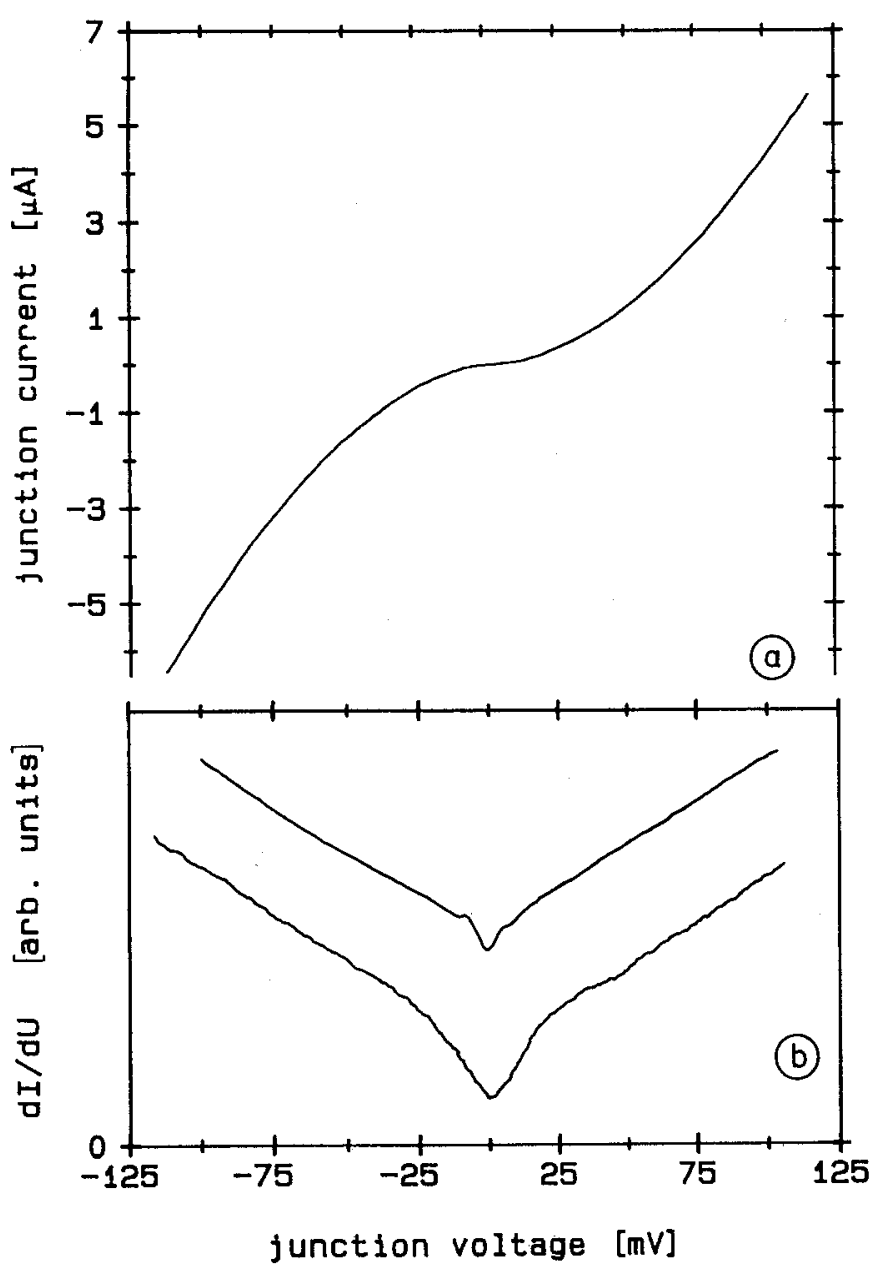

Fig. 1. Part a shows a typical $I-U$ curve for a high impedance tunneling contact between two pieces of $\mathrm{La}_{1.85} \mathrm{Sr}_{0.15} \mathrm{CuO}_{4}$ at $4.2 \mathrm{~K}$. No distinct step at $2 \Delta$ is observed. In b the derivative $\mathrm{d} I / \mathrm{d} U$ of this $I-U$ characteristic is shown as the upper curve, whereas the lower curve refers to a different contact spot on the sample. The energy gaps derived from these plots are $\Delta \approx 4 \mathrm{meV}$ and $10 \mathrm{meV}$, respectively

for these high ohmic contacts a minimum in the differential conductance around the origin at $U=0$, shown in Fig. 1, as it is known for single particle tunneling. The $I-U$ curve in Fig. 1 a does not display the features expected for a sharp gap, namely characteristic steps at voltage values of $U= \pm 2 \Delta / e$, where $\Delta$ is the energy gap of the superconductor and $e$ the electronic charge. Yet indications of a gap, or rather a distribution of gaps, are obtained when the differentiated characteristic, $\mathrm{d} I / \mathrm{d} U$, is considered in Fig. $1 \mathrm{~b}$. The two curves refer to separate spots on the same samples. Superimposed on a smooth background reflecting the overall $s$-shaped $I-U$ curve is a pronounced central dip, which started to appear only at tempera-

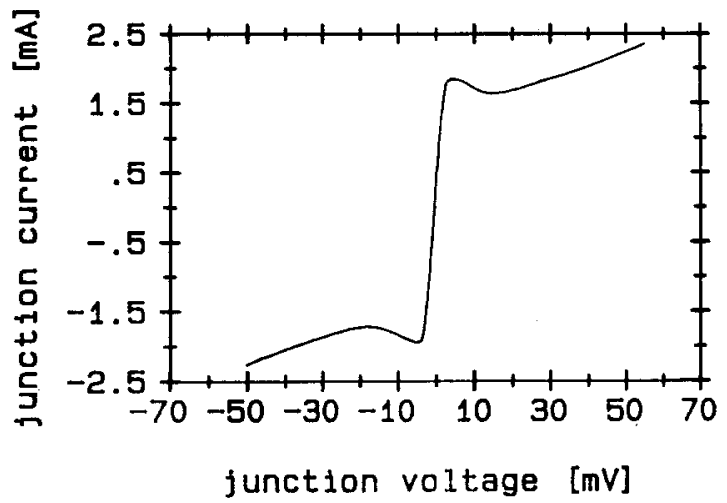

Fig. 2. $I-U$ characteristic of a low impedance contact at $4.2 \mathrm{~K}$. The supercurrent through the junction is limited by a critical value given by the height of the step

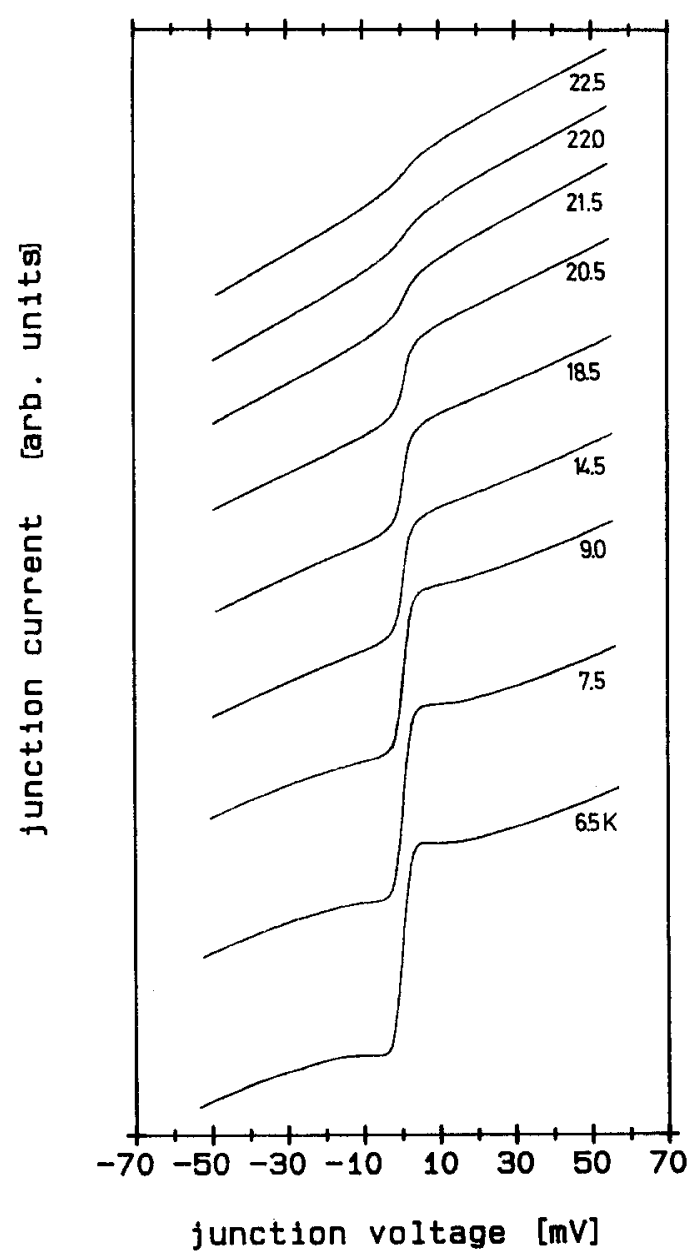

Fig. 3. $I-U$ characteristic of a low impedance contact at various temperatures. The decrease of the critical current with increasing temperature is evident

tures below roughly $30 \mathrm{~K}$. We interpret this feature as a signature of superconductivity (the bulk $T_{c}$ for these samples as determined from resistivity measure- 


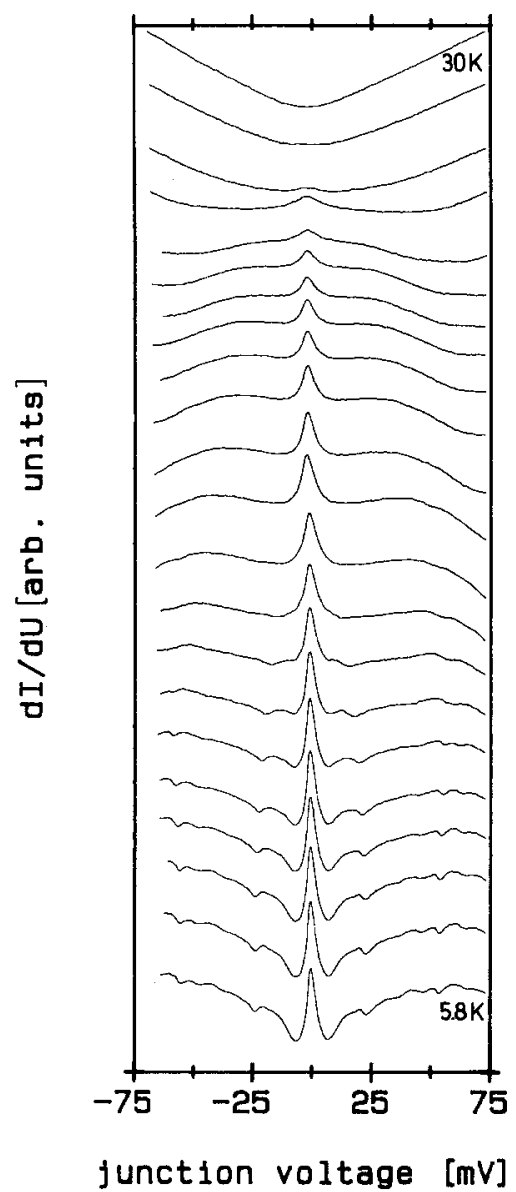

Fig. 4. $\mathrm{d} I / \mathrm{d} U$ curves of a point contact at various temperatures (from bottom to top: $5.8 ; 6.2 ; 6.5 ; 7.4 ; 8.1 ; 9.0 ; 10.8 ; 12.6 ; 14.5$; $15.5 ; 17.2 ; 18.6 ; 19.6 ; 20.5 ; 21.0 ; 21.5 ; 22.0 ; 23.4 ; 25.6 ; 28.0 ; 29.0$; $30.0 \mathrm{~K})$. The central peaks are due to the supercurrent through the contact. In addition to the critical current, proportional to the peak height at $U=0$, also the fine structure at $U \searrow 0$ reveals a temperature dependence

ments was $38 \mathrm{~K}$ ). The widths of both dips, which should correspond to $4 \Delta / e$, is $16 \mathrm{mV}$ and $40 \mathrm{mV}$, respectively. Such a scatter of the values for the gap $\Delta$ for different locations of the contacts was typical for our material, and even larger variations were observed for samples exposed to air for an extended period of time.

The $I-U$ curve for the low junction resistance corresponding to a small (and eventually vanishing) electrode spacing resembles the characteristics of a weak link or a point contact. In this case the $I-U$ curve rises sharply at $U=0$, provided the sample is superconducting. This is illustrated in Fig. 2. The plot shows the original trace of the $I-U$ characteristic, registered on a chart recorder without averaging. It demonstrates the quite satisfactory stability of the experimental set-up. The curve displays a pronounced jump in $I$ at $U=0$ due to the existence of a critical

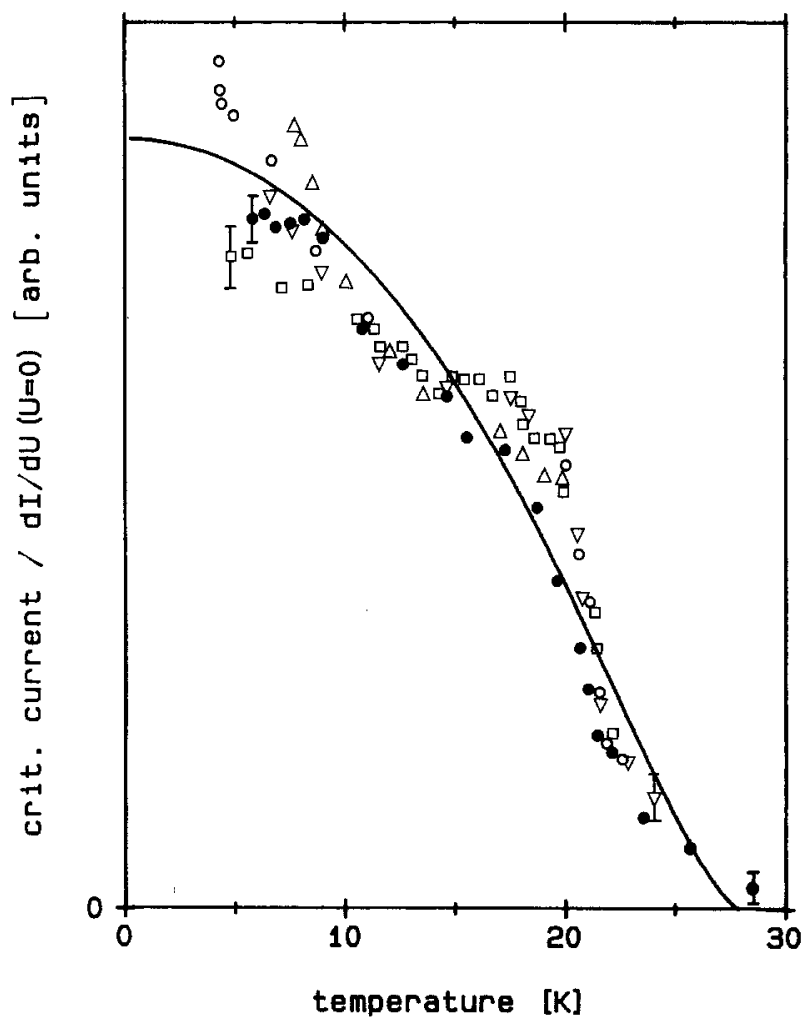

Fig. 5. Temperature dependence of the critical current through point contacts of $\mathrm{La}_{1.85} \mathrm{Sr}_{0.15} \mathrm{CuO}_{4}$. The open symbols refer to measurements of $I_{c}$ in several junctions like in Fig. 3, the closed symbols represent the critical current derived from the peak heights of $\mathrm{d} I / \mathrm{d} U$ in Fig. 4. The solid line shows the dependence calculated according to (3)

current $I_{c}$. A magnetic field of $\sim 10^{-2}$ Tesla had no noticeable influence on $I_{c}$.

When the temperature was raised, the critical current dropped and finally vanished in our samples around $30 \mathrm{~K}$. A sequence of $I-U$ curves illustrates this behavior in Fig. 3.

More details can be seen from the derivatives, as shown in Fig. 4 for a different contact. The supercurrent through the junction gives rise to a sharp peak at $U=0$ which vanishes as $T$ approaches the bulk transition temperature. The additional structures at finite voltage, which are evident at low temperatures, appear to merge into the zero voltage peak when the temperature increases. We attribute them to links in the current-carrying path which become normal when the current exceeds the local critical current. Since the width of the peak at $U=0$ is nearly constant, its height is a direct measure of $I_{c}$.

The temperature dependence of the critical current for several contacts is shown in Fig. 5, where we have plotted the jump in the $I-U$ characteristics as well as the peak heights in the derivatives. 


\section{Discussion}

The two curves of the derivative $\mathrm{d} I / \mathrm{d} U$ for the two characteristic tunnel junctions (Fig. $1 \mathrm{~b}$ ) demonstrate the inhomogeneity of the new high- $T_{c}$ superconductors. The maximum value for the BCS-ratio $2 \Delta / k_{B} T_{c}=6.1$ derived from the width $4 \Delta / e=40 \mathrm{mV}$ and a transition temperature of $38 \mathrm{~K}$ indicate that the new materials belong to the class of strong coupling superconductors. It should be mentioned that this ratio is rather high in comparison with other conventional superconductors [8], and it would be even larger, namely 7.7, if we assume that the local $T_{c}$ in the contact area is only $30 \mathrm{~K}$. Since a value of $2 \Delta / k_{B} T_{c} \approx 9.5$ is still consistent with electron-phonon coupling in the Eliashberg theory [9], a classical BCS-type superconductivity cannot be ruled out for these materials. Similar results, both regarding the absolute value of the gap as well as the considerable variation in the characteristics for different contacts of the same sample have been reported very recently $[10,11]$.

In order to interpret the jump in the $I-U$ characteristics we discuss as two possibilities first the critical current through a Josephson junction, and secondly a limit for the current given by the critical magnetic field:

i) The maximum critical current expected for a Josephson junction is given by [8]

$I_{c}=\pi \Delta / 2 e R_{n}$

where $R_{n}$ is the junction resistance in the normal state. The variation of $I_{c}$ with temperature is therefore dominated in this case by the temperature dependence of the energy gap of the superconductor. For a comparison with the critical current derived from Fig. 2 we take $R_{n}=20 \Omega$ and $\Delta / e=10 \mathrm{meV}$. The maximum current predicted by Eq. (1) is then $I_{c}=0.8 \mathrm{~mA}$, which is a factor of 3 smaller than the value observed in the experiment. This result, together with the fact that the current is not influenced by a small magnetic field leads us to the conclusion that the junctions of Figs. 2-4 were relatively strong contacts between the two superconductors rather than Josephson junctions. Hence Eq. (1) will not apply, in which case the data of Fig. 5 will not reflect the $T$-dependence of the energy gap.

ii) Another limit for a supercurrent is reached when the magnetic field in the junction due to the current exceeds the critical field $H_{c 2}$. The critical current will then primarily follow the T-dependence of the critical field. For a crude comparison with our data we con- sider the reduced critical current

$I_{c}(t) / I_{c}(0)=\left(H_{c}(t) / H_{c}(0)\right) /(\lambda(t) / \lambda(0))$

as it is obtained for example for a thin wire [12]. Here $\lambda$ is the penetration depth of the magnetic field and $t=T / T_{c}$. The calculation using the usual (type I superconductor) temperature dependences yields

$I_{c}(t) / I_{c}(0)=\left(1-t^{2}\right)^{2 / 3}\left(1+t^{2}\right)^{1 / 2}$

which we have plotted as the full line in Fig. 5, assuming for $T_{c}$ a value of $28 \mathrm{~K}$. Although the gross shape is reproduced, the data show additional structure around $20 \mathrm{~K}$, which is not accounted for even if more realistic estimates for the $T$-dependences of $H_{c 2}$ are used [13]. A possible origin are inhomogeneities of the sample.

\section{Conclusions}

The $I-U$ characteristics of small contacts between two pieces of the high- $T_{c}$ superconductor $\mathrm{La}_{1.85} \mathrm{Sr}_{0.15} \mathrm{CuO}_{4}$ reveal structures which yield information about the energy gap and, for the first time, about the local critical currents in this material. The results support the assumption that these metal oxides belong to the class of strong coupling superconductors. The broad distribution of energy gaps for different contacts on the same sample indicate the inhomogeneity of the present samples. The critical currents measured in our experiment appear to be dominated by the local critical field $H_{c 2}$.

We appreciate useful discussions with the members of Sonderforschungsbereich SFB 252 as well as financial support by the Deutsche Forschungsgemeinschaft.

\section{References}

1. Bednorz, J.G., Müller, K.A.: Z. Phys. B - Condensed Matter 64, 189 (1986)

2. Chu, C.W., Hor, P.H., Meng, R.L., Gao, L., Huang, Z.J., Wang, Y.Q.: Phys. Rev. Lett. 58, 405 (1987)

3. Takagi, H., Uchida, S., Kitizawa, K., Tanaka, S.: (to be published)

4. Cava, R.J., Dover, R.B. van, Batlogg, B., Rietmann, E.A.: Phys. Rev. Lett. 58, 408 (1987)

5. Politis, C., Geerk, J., Dietrich, M., Obst, B.: Z. Phys. B - Condenset Matter 66, 141 (1987)

6. Renker, B., Gompf, F., Gering, E., Nücker, N., Ewert, D., Reichardt, W., Rietschel, H.: Z. Phys. B - Condensed Matter 67, 15 (1987)

7. Feile, R., Leiderer, P.: Post-deadline contribution at the Spring 
Meeting of the Deutsche Physikalische Gesellschaft, Münster, March 1987

8. Wolf, E.L.: Principles of electron tunneling spectroscopy. New York: Oxford University Press 1985

9. Carbotte, J.P., Marsiglio, F., Mitrovic, B.: Phys. Rev. B33, 6135 (1986)

10. Moreland, J., Clark, A.F., Ku, H.C., Shelton, R.N.: (to be published)

11. Hawley, M.E., Gray, K.E., Capone, D.W., Hinks, D.G.: (to be published)

12. Newhouse, V.L.: Applied superconductivity. New York: John Wiley \& Sons 1964

13. See e.g. Fetter, A.L., Hohenberg, P.C.: In: Superconducitivity. Parks, R.P. (eds.), Vol. II, p. 817. New York: Marcel Dekker 1969
P. Leiderer, R. Feile

Institut für Physik

Johannes Gutenberg-Universität

Postfach 3980

D-6500 Mainz 1

Federal Republic of Germany

B. Renker, D. Ewert

Institut für Nukleare Festkörperphysik

Kernforschungszentrum Karlsruhe $\mathrm{GmbH}$

Postfach 3640

D-7500 Karlsruhe 1

Federal Republic of Germany 